\title{
Confidence-Related Decision Making
}

\author{
Andrea Insabato, ${ }^{1}$ Mario Pannunzi, ${ }^{1}$ Edmund T. Rolls, ${ }^{2}$ and Gustavo Deco ${ }^{1}$ \\ ${ }^{1}$ Theoretical and Computational Neuroscience, Center for Brain and Cognition, Universitat Pompeu Fabra, and Institució Catalana de \\ Recerca i Estudis Avancats, Barcelona, Spain; and ${ }^{2}$ Oxford Centre for Computational Neuroscience, Oxford, United Kingdom
}

Submitted 8 December 2009; accepted in final form 12 April 2010

\begin{abstract}
Insabato A, Pannunzi M, Rolls ET, Deco G. Confidence-related decision making. J Neurophysiol 104: 539-547, 2010. First published April 14, 2010; doi:10.1152/jn.01068.2009. Neurons have been recorded that reflect in their firing rates the confidence in a decision. Here we show how this could arise as an emergent property in an integrate-and-fire attractor network model of decision making. The attractor network has populations of neurons that respond to each of the possible choices, each biased by the evidence for that choice, and there is competition between the attractor states until one population wins the competition and finishes with high firing that represents the decision. Noise resulting from the random spiking times of individual neurons makes the decision making probabilistic. We also show that a second attractor network can make decisions based on the confidence in the first decision. This system is supported by and accounts for neuronal responses recorded during decision making and makes predictions about the neuronal activity that will be found when a decision is made about whether to stay with a first decision or to abort the trial and start again. The research shows how monitoring can be performed in the brain and this has many implications for understanding cognitive functioning.
\end{abstract}

\section{IN T R O D U C T I O N}

Decision confidence - the feeling of having done something correctly or incorrectly-is an important aspect of subjective experience during decision making, which increases for correct decisions and decreases for error decisions as the task become easier (Jonsson et al. 2005; Vickers 1979; Vickers and Packer 1982). Neurons in the macaque parietal cortex involved in perceptual decision making about motion stimuli have firing rates that are higher for decisions in which there is confidence (Kiani and Shadlen 2009). Kepecs and colleagues (2008) described neurons in the rat orbitofrontal cortex (OFC) as encoding decision confidence in an olfactory classification task. In the task, a mixture of odors A and B was categorized as $\mathrm{A}$ or B, depending on which odor was predominant. The difficulty of the task could be controlled by varying the proportion of the two odors. A second experiment was undertaken to examine the ability of the rats to behave in accordance with the confidence-related information. In this second experiment the rat had the possibility of aborting the current trial without waiting for the reward outcome and thus starting a new trial. The results showed that a second decision (about whether to abort the trial) could be made based on confidence in the first decision, in that the rats were more likely to abort a trial if they had made an error in the odor classification. Two different decisions should then be distinguished: a first-stage decision (about the stimulus) and a second-stage decision based on the level of confidence in the first decision.

Address for reprint requests and other correspondence: E. T. Rolls, Oxford Centre for Computational Neuroscience, Oxford, UK (E-mail: Edmund. Rolls@oxcns.org; url:www.oxcns.org).
Here we propose an integrate-and-fire attractor network model (shown in Fig. 1), to account for decision confidence mechanisms (and described in detail in the Supplemental material). "This is a "mechanistic" biologically realistic approach and not a "phenomenological" approach such as an accumulator or race model that accumulates noisy evidence with a linear integrator until some threshold is reached (Gold and Shadlen 2007; Ratcliff and Rouder 1998; Ratcliff et al. 1999; Usher and McClelland 2001; Vickers and Packer 1982). We show that decision confidence is an emergent property of decision-making neural networks (for well-known implementations see Deco and Rolls (2006); Wang (2002)) encoded in the firing rates of the neurons (the first module in Fig. 1). However, this does not account for subsequent confidencebased decisions as in the second experiment of Kepecs et al. (2008). For this we propose that a second decision-making network is needed (the second module in Fig. 1). Thus our main proposal is for a two-layer model of confidence-related decision making, which can account for the two decisionmaking processes and elucidates how confidence-related decision-making mechanisms could operate in the brain. We analyze how such a system would work, analyze its properties, and show that it accounts for the neurophysiological results described by Kepecs et al. (2008). Moreover, the model leads to predictions about new properties of these neurons that can be tested.

\section{METHODS}

\section{Neuron and synapse model}

At the neuronal level we used single-compartment leaky integrateand-fire (IF) neurons that incorporate biophysically realistic parameters (Abeles 1991) without being computationally intractable (Brunel and Wang 2001; Dayan and Abbott 2001; Deco and Rolls 2006; Wang 2002). The integrate-and-fire formulation is very important because the spiking of the neurons in the network is close to random in its timing for a given mean firing rate (Poisson-like), which introduces noise into the network that enables it to account for probabilistic decision making because the choices are influenced by the spikingrelated coherent statistical fluctuations that are produced in a finitesize network.

The dynamics of the membrane potential in the subthreshold regime is determined by the membrane capacitance and a leakage term, following the equation

$$
C_{\mathrm{m}} \dot{V}(t)=-g_{L}\left(V(t)-V_{L}\right)-I_{\text {syn }}(t)
$$

where $C_{\mathrm{m}}$ is the membrane capacitance, $g_{\mathrm{L}}$ is the membrane conductance that provides the leakage current, $V_{\mathrm{L}}$ is the resting potential, and $I_{\text {syn }}$ is the total input synaptic current. When the depolarization of the membrane reaches a threshold value $V_{\theta}$, the neuron fires a spike, its

\footnotetext{
${ }^{1}$ The online version of this article contains supplemental data.
} 


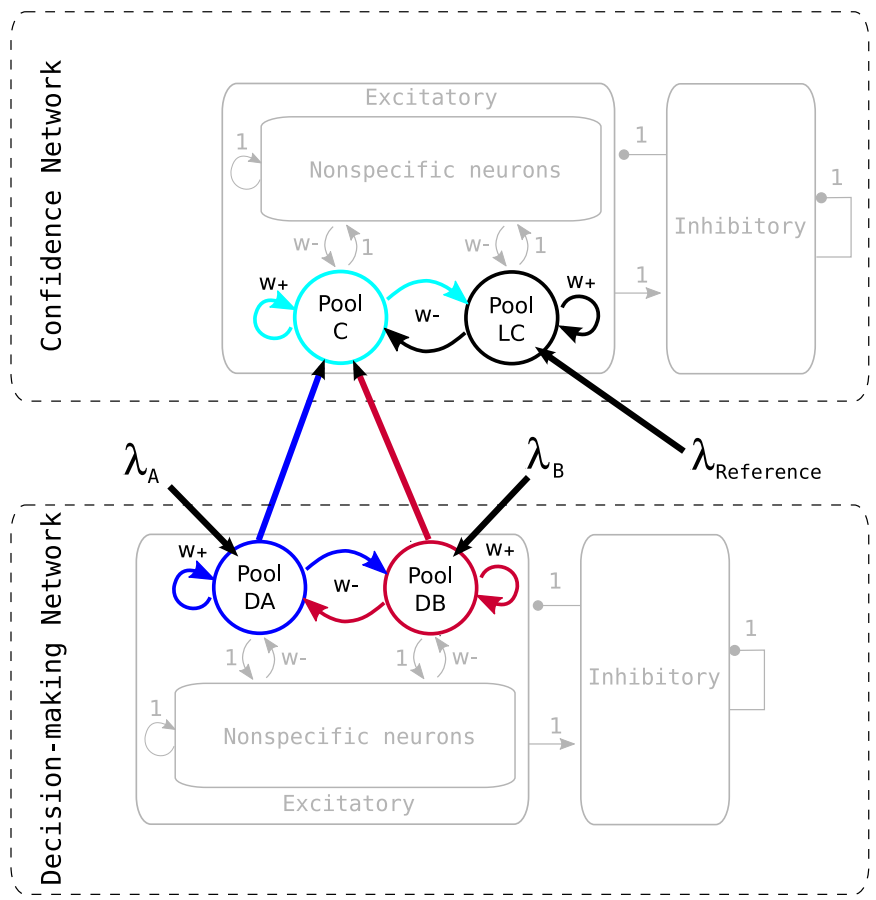

FIG. 1. Network architecture for decisions about confidence estimates. The first network is a decision-making network and its outputs are sent to a second network that makes decisions based on the firing rates from the first network, which reflect the decision confidence. In the first network, high firing of neuronal population (or pool) DA represents decision A and high firing of population DB represents decision $\mathrm{B}$. Pools DA and DB receive a stimulusrelated input ( $\lambda_{\mathrm{A}}$ and $\lambda_{\mathrm{B}}$, respectively), the evidence for each of the decisions, and these bias the attractor networks, which have internal positive feedback produced by the recurrent excitatory connections. Pools DA and DB compete through inhibitory interneurons. The neurons are integrate-and-fire spiking neurons with random spiking times (for a given mean firing rate), which introduce noise into the network and influence the decision making, making i probabilistic. The second network is a confidence decision attractor network and receives inputs from the first network. The confidence network has two selective pools of neurons, one of which (C) responds to represent confidence in the decision and the other of which responds when there is little or a lack of confidence in the decision (LC). The $\mathrm{C}$ neurons receive the outputs from the selective pools of the (first) decision-making network and the LC neurons receive $\lambda_{\text {Reference }}$, which saturates at 40 spikes/s, a rate that is close to the rates averaged across correct and error trials of the sum of the firing in the selective pools in the (first) decision-making network. In each network the excitatory pool is divided into 3 subpopulations: a nonspecific one and two stimulusselective populations. Each selective pool has strong recurrent connections $\left(w_{+}\right)$, whereas the connections between the 2 selective pools are weak $\left(w_{-}\right)$. All other connections are set to the default value 1 .

voltage is reset to a given value $V_{\text {reset }}$, and a refractory period $\tau_{\text {rp }}$ follows during which the neuron is unable to fire another spike.

The total synaptic current is the sum of the external excitatory currents mediated by $\alpha$-amino-3-hydroxy-5-methyl-4-isoxazolepropionic acid (AMPA) receptors $\left(I_{\mathrm{AMPA}, \mathrm{ext}}\right)$, recurrent excitatory currents mediated by both AMPA receptors $\left(I_{\text {AMPA,rec }}\right)$ and $N$-methyl-D-aspartate (NMDA) receptors $\left(I_{\mathrm{NMDA}}\right)$, and inhibitory currents mediated by $\gamma$-aminobutyric acid $(\mathrm{GABA})$ receptors $\left(I_{\mathrm{GABA}}\right)$

$$
I_{\mathrm{syn}}(t)=I_{\mathrm{AMPA}, \mathrm{ext}}(t)+I_{\mathrm{AMPA}, \mathrm{rec}}(t)+I_{\mathrm{NMDA}}(t)+I_{\mathrm{GABA}}(t)
$$

Each current is defined by

$$
\begin{gathered}
I_{\text {AMPA,ext }}(t)=g_{\text {AMPA,ext }}\left(V(t)-V_{E}\right) \sum_{j=1}^{N_{\text {ext }}} s_{j}^{\mathrm{AMPA}, \mathrm{ext}}(t) \\
I_{\mathrm{AMPA}, \mathrm{rec}}(t)=g_{\text {AMPA,rec }}\left(V(t)-V_{E}\right) \sum_{j=1}^{N_{E}} w_{j} s_{j}^{\mathrm{AMPA,rec}}(t)
\end{gathered}
$$

$$
\begin{gathered}
I_{\mathrm{NMDA}}(t)=\frac{g_{\mathrm{NMDA}}\left(V(t)-V_{E}\right)}{1+\left[\mathrm{Mg}^{2+}\right] \exp (-0.062 V(t)) / 3.57} \times \sum_{j=1}^{N_{\mathrm{E}}} w_{j} s_{j}^{\mathrm{NMDA}}(t) \\
I_{\mathrm{GABA}}(t)=g_{\mathrm{GABA}}\left(V(t)-V_{I}\right) \sum_{j=1}^{N_{I}} w_{j} s_{j}^{\mathrm{GABA}}(t)
\end{gathered}
$$

where $g_{\text {AMPA,ext }}, g_{\text {AMPA,rec }}, g_{\mathrm{NMDA}}$, and $g_{\mathrm{GABA}}$ are the receptor-specific synaptic conductances; $V_{\mathrm{E}}$ and $V_{\mathrm{I}}$ are the reversal potentials, respectively, for the excitatory and inhibitory neurons; $N_{\text {ext }}, N_{\mathrm{E}}$, and $N_{\mathrm{I}}$ are, respectively, the number of external neurons residing in other cortical areas, of excitatory neurons and of inhibitory neurons; $s_{j}^{\text {AMPA,ext }}, s_{j}^{\text {AMPA,rec }}$, $s_{j}^{\mathrm{NMDA}}$, and $s_{j}^{\mathrm{GABA}}$ are the receptor-specific fractions of open channels; and $w_{j}$ are the synaptic weights, defining the pools. The NMDA receptoractivated synaptic currents are dependent on the membrane voltage and are controlled by the extracellular concentration of magnesium $\left[\mathrm{Mg}^{2+}\right]$. The fraction of open channels is given by

$$
\begin{gathered}
\frac{\mathrm{d} s_{j}^{\mathrm{AMPA}, \mathrm{ext}}(t)}{\mathrm{d} t}=-\frac{s_{j}^{\mathrm{AMPA}, \mathrm{ext}}(t)}{\tau_{\mathrm{AMPA}}}+\sum_{k} \delta\left(t-t_{j}^{k}\right) \\
\frac{\mathrm{d} s_{j}^{\mathrm{AMPA}, \mathrm{rec}}(t)}{\mathrm{d} t}=-\frac{s_{j}^{\mathrm{AMPA}, \mathrm{rec}}(t)}{\tau_{\mathrm{AMPA}}}+\sum_{k} \delta\left(t-t_{j}^{k}\right) \\
\frac{\mathrm{d} s_{j}^{\mathrm{NMDA}}(t)}{\mathrm{d} t}=-\frac{s_{j}^{\mathrm{NMDA}}(t)}{\tau_{\mathrm{NMDA}, \mathrm{decay}}}+\alpha x_{j}(t)\left(1-s_{j}^{\mathrm{NMDA}}(t)\right) \\
\frac{\mathrm{d} x_{j}^{\mathrm{NMDA}}(t)}{\mathrm{d} t}=-\frac{x_{j}^{\mathrm{NMDA}}(t)}{\tau_{\mathrm{NMDA}, \mathrm{rise}}}+\sum_{k} \delta\left(t-t_{j}^{k}\right) \\
\frac{\mathrm{d} s_{j}^{\mathrm{GABA}}(t)}{\mathrm{d} t}=-\frac{s_{j}^{\mathrm{GABA}}(t)}{\tau_{\mathrm{GABA}}}+\sum_{k} \delta\left(t-t_{j}^{k}\right)
\end{gathered}
$$

where $\tau_{\text {AMPA,ext }}, \tau_{\text {AMPA,rec }}, \tau_{\text {NMDA,decay }}$, and $\tau_{\text {GABA }}$ are the decay time constants and $\tau_{\text {NMDA,rise }}$ is the rise time constant for NMDA synapses. The rise times for AMPA and GABA synapses are neglected because they are $<1 \mathrm{~ms}$. The sums over $k$ represent the sums over spikes formulated as $\delta$-peaks $[\delta(t)]$ emitted by presynaptic neuron $j$ at time $k$.

The values for the neuronal and synaptic dynamics are provided in

\begin{tabular}{|c|c|c|c|}
\hline Parameter & Value & Parameter & Value \\
\hline$C_{\mathrm{m}}$ (excitatory) & $0.5 \mathrm{nF}$ & $V_{\mathrm{E}}$ & $0 \mathrm{mV}$ \\
\hline$C_{\mathrm{m}}$ (inhibitory) & $0.2 \mathrm{nF}$ & $V_{\mathrm{I}}$ & $-70 \mathrm{mV}$ \\
\hline$f$ & 0.15 & $V_{\mathrm{L}}$ & $-70 \mathrm{mV}$ \\
\hline$g_{\text {AMPA, ext }}$ (excitatory) & $2.08 \mathrm{nS}$ & $V_{\text {reset }}$ & $-55 \mathrm{mV}$ \\
\hline$g_{\text {AMPA,ext }}$ (inhibitory) & $1.62 \mathrm{nS}$ & $V_{\theta}$ & $-50 \mathrm{mV}$ \\
\hline$g_{\text {AMPA,rec }}$ (excitatory) & $0.104 \mathrm{nS}$ & $\begin{array}{l}w_{+}(\text {decision-making } \\
\text { network) }\end{array}$ & 1.8 \\
\hline$g_{\text {AMPA, rec }}$ (inhibitory) & $0.081 \mathrm{nS}$ & $w_{+}$(confidence network) & 1.7 \\
\hline$g_{\mathrm{GABA}}($ excitatory) & $1.287 \mathrm{nS}$ & $\alpha$ & $0.5 \mathrm{~ms}^{-1}$ \\
\hline$g_{\mathrm{GABA}}$ (inhibitory) & $1.002 \mathrm{nS}$ & $\lambda_{\text {Reference }}$ & $40 \mathrm{~Hz}$ \\
\hline$g_{\text {NMDA }}$ (excitatory) & $0.327 \mathrm{nS}$ & $\lambda_{\text {ext }}$ & $2.4 \mathrm{kHz}$ \\
\hline$\lambda$ & $45 \mathrm{~Hz}$ & $\Delta \lambda$ & {$\left[\begin{array}{ll}0 & 30\end{array}\right] \mathrm{Hz}$} \\
\hline$g_{\mathrm{NMDA}}$ (inhibitory) & $0.258 \mathrm{nS}$ & $\tau_{\mathrm{AMPA}}$ & $2 \mathrm{~ms}$ \\
\hline$N_{\mathrm{E}}$ & 800 & $\tau_{\mathrm{GABA}}$ & $10 \mathrm{~ms}$ \\
\hline$N_{\mathrm{I}}$ & 200 & $\tau_{\mathrm{NMDA}, \text { decay }}$ & $100 \mathrm{~ms}$ \\
\hline$N_{\text {ext }}$ & 800 & $\tau_{\mathrm{NMDA}, \text { rise }}$ & $2 \mathrm{~ms}$ \\
\hline
\end{tabular}
Table 1. We implemented synaptic dynamics and, although not essential for the model, included in them the slow synaptic dynamics produced by the long time constant of the NMDA receptors because, as shown by Wang (1999), to stabilize the network the recurrent excitation should be dominated by slow synaptic dynamics, such as those produced by the NMDA receptors. Moreover, we used these synaptic dynamics to avoid synchrony and oscillations, following Brunel and Wang (2003). We did not implement synaptic delays for

\section{TABLE 1. Default parameters used in the simulations}


simplicity. Transmission delays help to prevent synchrony in the network (Mattia and Del Giudice 2003), producing similar effects in this respect to slow synaptic dynamics (implemented in the NMDA receptors). Since we used NMDA receptors, we expect our results to remain valid without explicitly implementing synaptic delays.

\section{Parameter setting}

We use a mean-field reduction (Brunel and Wang 2001) to study the space of the principal parameters of each network. All other parameters were set according to the results of Marti and colleagues (2006). Details of the mean-field analysis are given in the Supplemental materials. Once the parameters of the two separate modules had been set, we chose the values of the intermodule connections to be the same as those of the standard connections from the external inputs. All parameter values are reported in Table 1.

Once the parameters were fixed using the mean-field analysis we ran spiking simulations. Both the mean-field reduction and spiking simulations were implemented in custom $\mathrm{C}++$ programs. For the mean-field numerical integration we used a Euler routine with a step size of 0.1 . For the spiking simulations we used a second-order Runge-Kutta routine, with a time step of $0.02 \mathrm{~ms}$, to perform numerical integration of the coupled differential equations that describe the dynamics of all cells and synapses. The population firing rates were calculated by performing a spike count over a $50 \mathrm{~ms}$ window moved with a time step of $5 \mathrm{~ms}$. This sum was then divided by the number of neurons in the population and by the window size.

\section{R E S U L T S}

\section{The model: network architecture}

Our model is composed of two modules. Each one is an attractor neural network implementing a decision-making process. The first module is designed to make a perceptual decision (odor classification). We will refer to it as the decisionmaking (first) network. The second module, which receives inputs from the decision-making network, "decides" whether to abort the task, estimating the level of confidence in the first perceptual decision. We will refer to it as the confidence decision-making (second) network (see Fig. 1).

The simple scheme of a neural network implementing a decision-making process, developed by Wang (2002), is composed of two selective pools of excitatory neurons, one nonselective pool of excitatory neurons, and one pool of inhibitory neurons. The nonselective pool represents the background activity of the neurons not responding to the stimulus. The two selective pools represent the choices. The neurons within each excitatory selective population have strong recurrent connections and there are weak connections between the pools. When an external input is delivered to one or both of the selective pools the activity increases, causing an enhancement of the inhibition. Since the two pools are mutually connected they cooperate in increasing the activity. When the inhibitory current is sufficiently strong a competition takes place between the selective pools. One of the two pools wins the competition and ends up with a high firing rate, whereas the other pool ends up with a low firing rate, indicating that a decision state is reached. The balance between competition and cooperation depends principally on the parameters of the mutual connections between the pools and the recurrent connections within a pool. The evidence for each decision is applied as an external excitatory input to each population of neurons and biases the competition in favor of one of the two pools. The arriving random spike trains (with a Poissonian spike time distribution) together with finite-size effects produce the stochastic dynamics of the network and the probabilistic decision making, as described in more detail elsewhere (Deco and Marti 2007; Deco et al. 2009; Marti et al. 2008; Rolls and Deco 2010).

The two selective pools of the decision-making network are DA and DB, which become active for decisions $\mathrm{A}$ and $\mathrm{B}$, respectively. During the stimulation, pool DA (DB) receives sensory information about odor A (or B) via external input $\lambda_{\mathrm{A}, \mathrm{B}}$. When stimulus A (or B) is applied, pool DA (or DB) will usually win the competition and end up with high firing, indicating that decision A (or B) has been reached. When a mixture is applied, the decision-making network will probabilistically choose DA or DB, influenced by the proportion of $\mathrm{A}$ and $\mathrm{B}$ in the mixture.

The parameters of the decision-making (first) network were chosen as follows. The inputs to the network from the sensory stimuli A and B and the synaptic weights between the neurons within a pool were set so that the network operated in a bistable regime, by which we mean that the spontaneous state is no longer stable when the decision cues are being applied. Then noise and sensory inputs bias the landscape and provoke a transition to a decision state. Thus the attractor basins are influenced by the stimulus. Whether the network can present persistent activity in the absence of stimulation is not important for our purpose. In addition the inputs were set so that with only one stimulus in the mixture, the decision was roughly $100 \%$ correct. Details of the mean-field analytic approach are provided in the Supplemental material.

The confidence network has two selective pools of neurons, one of which $(\mathrm{C})$ responds to indicate confidence in the first decision and to stay with the first decision based on a level of firing from the first network, which indicates high confidence, and the other of which (LC) responds when there is little or a lack of confidence in the first-stage decision. In the experiment of Kepecs et al. (2008), C corresponds to a decision to stay and wait for a reward, i.e., what they call the positive outcome population, although it really represents confidence or a prediction that the decision just made will have a positive outcome. LC corresponds to a second decision to abort a trial and not wait for a possible reward, i.e., what they call the negative outcome population, although it really represents lack of confidence that the perceptual decision just made will have a positive outcome, equivalent to confidence that the decision just made will have a negative outcome.

The two networks are connected by AMPA synapses that link the selective pools DA and DB of the (first) decisionmaking network to confidence network selective pool C. (The synaptic conductances of these connections are set to the value $g_{\text {AMPA,ext }}=2.08 \mathrm{nS}$, although this value is not crucial for the mechanism.) The selective pool LC in the confidence network receives an external input that saturates at $40 \mathrm{spikes} / \mathrm{s}$, to set the competition with pool $\mathrm{C}$. This input could come from the same source as that to the $\mathrm{C}$ network, or could come from other brain areas, and could reflect the subject's bias in his or her confidence.

The total number of neurons in the model is $N=2,000$. For simplicity we chose to have the same number of neurons in each of the two networks: $N_{\text {mod }}=N / 2$. Therefore each network has $N_{\mathrm{E}}=0.8 \cdot N_{\text {mod }}$ excitatory pyramidal neurons and $N_{\mathrm{I}}=0.2 \cdot N_{\text {mod }}$ inhibitory interneurons, the proportions observed in the cerebral 
cortex (Abeles 1991). The number of neurons in each selective pool is $N_{\mathrm{A}}=N_{\mathrm{B}}=N_{\mathrm{C}}=N_{\mathrm{LC}}=N_{\mathrm{E}} \cdot f$, where $f$ is the fraction of excitatory neurons in each selective pool. In this study we set $f=0.15$. Each nonspecific pool in each network contains the remaining $N_{\mathrm{E}}-N_{\mathrm{A}}-N_{\mathrm{B}}$ excitatory neurons. We modeled an equal number of neurons in each selective pool to keep the model as simple as possible and note that equal numbers of neurons for the different attractors need not be present in this class of attractor network (Rolls 2008). Each network is fully connected-i.e., all neurons are connected to each other. We note that sparse connectivity does not change the overall dynamics of the network (i.e., the mechanism is also reproducible with a sparsely connected network), bringing about merely an increase of the noise in the network due to the finite-size effect (Mattia and Del Giudice 2002, 2004). We make the plausible hypothesis that the connection strengths have been modified from their default value of 1 by a previous learning process and thus we set them following a Hebb-like rule: i.e., the synaptic efficacy between two cells is high if the cells had correlated activity in the past, whereas uncorrelated activity results in a weak synapse. Cells in one selective pool have strong recurrent connections $w_{+}$, whereas synaptic efficacy between the two selective pools is decreased, given by $w_{-}=\left(1-f w_{+}\right) /(1-f)$. We set these parameters $\left(w_{+}, w_{-}\right)$to slightly different values for the two modules. To achieve a better correspondence with the results of Kepecs et al. (2008) we used a weaker $w_{+}$in the confidence network than that in the decision-making network, as shown in Table 1. During the simulation all the synaptic weights are kept fixed. All neurons receive an external input $\lambda_{\text {ext }}$, modeled as Poisson spike trains, from 800 external neurons each firing at a rate of $3 \mathrm{~Hz}$, consistent with observed values in the cortex. During the stimulation the external input changes for the selective pools as described earlier.

Our model has a two-stage structure and assumes some type of connectivity between the two modules or stages: the decision-making network and confidence network. These modules could both be within the OFC or they could be in different brain regions.

In the Supplemental material we describe the details of the mean-field approximation used to determine the parameters of the synaptic strengths in the model to obtain stable operation. Other details of the architecture that was implemented are reported in Table 2 and shown in Fig. 1.

\section{Simulation results}

Once the parameter values had been determined using the mean-field analysis, we ran simulation trials with these values, increasing the bias value, which corresponds to altering the stimulus identity, which in this case corresponds to altering the proportion of the two odors in the mixture. On each trial the network received for the first $500 \mathrm{~ms}$ the external spontaneous firing level input $\lambda_{\text {ext }}$. With this input the only stable state of the network is the spontaneous activity. After that, each selective pool DA and pool DB received in addition to $\lambda_{\text {ext }}$ the stimulus input $\lambda_{\mathrm{A}, \mathrm{B}}=\lambda \pm \Delta \lambda$, which provides the stimulus-specific information for the decision and drives the network dynamics. We ran different sets of simulations with $\Delta \lambda$ values of 0,10 , 20 , and $30 \mathrm{~Hz}$. We considered that a decision was reached when the selectivity index $S=\left|\ln v_{\mathrm{A}} / v_{\mathrm{B}}\right|$ took a value $>1.7$ and did not decrease for $\geq 100 \mathrm{~ms}$, where $v_{\mathrm{A}}$ and $v_{\mathrm{B}}$ are the firing rates of pools $\mathrm{A}$ and $\mathrm{B}$. The same criterion was used to determine the decision in the second network using the firing rates of the $\mathrm{C}$ and $\mathrm{LC}$ pools. Pool $\mathrm{C}$ received the external input and the output of pools DA and DB. Pool LC received just external input for $700 \mathrm{~ms}$ and after that an additional input, modeled as a Poisson process of mean rate $40 \mathrm{~Hz}$. We maintained the stimulation throughout the trial. This does not correspond to the experimental paradigm of Kepecs et al. (2008), although we hypothesized a working memory process upstream like the one described by Brody et al. (2003) and Machens et al. (2005).

First, we show how the firing rates of the (first) decisionmaking network reflect decision confidence. Figure $2 C$ shows the proportion of correct perceptual decisions as a function of the proportion of stimulus A and stimulus B in the mixture. The decision making is probabilistic because of the spikingrelated randomness in the network (Deco and Rolls 2006; Rolls and Deco 2010; Wang 2002). Figure $2 A$ shows that on trials when the DA neuronal population that represents decision A correctly wins and has a high firing rate, the firing rate increases further with the discriminability of the stimuli $\Delta \lambda$ and thus encodes increasing confidence. The reason for the increase of firing rate with $\Delta \lambda$ on correct trials is that the external inputs from stimulus A or stimulus B then support the (noise-influenced) winning attractor (pool DA) and add to the firing rates being produced by the recurrent collateral connections in the winning attractor. On the other hand, on error trials the firing rates of the winning pool (now DB, which represents decision $\mathrm{B}$ and wins despite the evidence because of noisy firing in the network) become lower as $\Delta \lambda$ increases because then the external sensory inputs are inconsistent with the perceptual decision that has been made and do not support and increase the firing rate of the winning pool (Rolls and Deco 2010; E. T. Rolls, F. Grabenhorst, and G. Deco, unpublished data). This modulation by stimulus difficulty of the firing rates of the decision-making populations was also observed experimentally by Felsen and Mainen (2009) in the superior colliculus. Confidence, which increases with $\Delta \lambda$ on correct trials and decreases with $\Delta \lambda$ on error trials (Jonsson et al. 2005; Kepecs et al. 2008; Vickers 1979; Vickers and Packer 1982; Rolls et al., unpublished data), is thus encoded in the firing rates of the winning attractor and is an emergent property of the decisionmaking network because it was not directly implemented in the model, but arises from the simple decision process (Rolls and Deco 2010; Wang 2002).

Moreover, the sum of the activity of the winning and losing populations also represents decision confidence on correct and error trials, as shown in Fig. 2B. It is this total firing from pools DA and DB of the first, decision-making, network that reflects decision confidence, which is provided as the input to the confidence (second) network.

We now consider the operation of the confidence decision (second) network. If the firing rate of the winning attractor of the first, decision-making, network is high, then the confidence decision network acting as a second-level network makes the second-stage decision, probabilistically as before, to have confidence in the first-stage decision and the $\mathrm{C}$ population probabilistically wins the competition. If the output firing of DA and DB (reflected in their sum) is low because the perceptual decision just made has sensory inputs that are not consonant with the decision, then with weaker driving inputs to the $\mathrm{C}$ 
TABLE 2. Neuron and synapse model

A. Model Summary

\begin{tabular}{l} 
Populations \\
Topology \\
Connectivity \\
Neuron model \\
Channel models \\
Synapse model \\
Plasticity \\
Input \\
Measurements \\
\hline \\
\hline Total number of neurons \\
Excitatory neurons in each module \\
Inhibitory neurons in each module \\
Name \\
DA (decision A) \\
DB (decision B) \\
C (confidence) \\
LC (lack of confidence)
\end{tabular}

Eight

Two modules partially connected

Full, no synaptic delay

Leaky integrate-and-fire (IF), fixed threshold, fixed refractory time

Instantaneous jump and exponential decay for AMPA and GABA and exponential jump and decay for NMDA receptors

Independent fixed-rate Poisson spike trains to all neurons

Spike activity

B. Populations

$$
\begin{aligned}
& N=2,000 \\
& N_{\mathrm{E}}=0.8 \cdot N_{\text {mod }} \\
& N_{\mathrm{I}}=0.2 \cdot N_{\text {mod }} \\
& \quad \text { Size } \\
& N_{\mathrm{A}}=f \cdot N_{\mathrm{E}} \\
& N_{\mathrm{B}}=f \cdot N_{\mathrm{E}} \\
& N_{\mathrm{C}}=f \cdot N_{\mathrm{E}} \\
& N_{\mathrm{LC}}=f \cdot N_{\mathrm{E}}
\end{aligned}
$$

In each module

$N_{\text {mod }}=N / 2$

Name

Nonspecific (1st module)

Inhibitory (1st module)

Nonspecific (2nd module)

Inhibitory (2nd module)

\section{Type}

Subthreshold dynamics

Synaptic currents

Fraction of open channels

Spiking

C. Neuron and Synapse Model

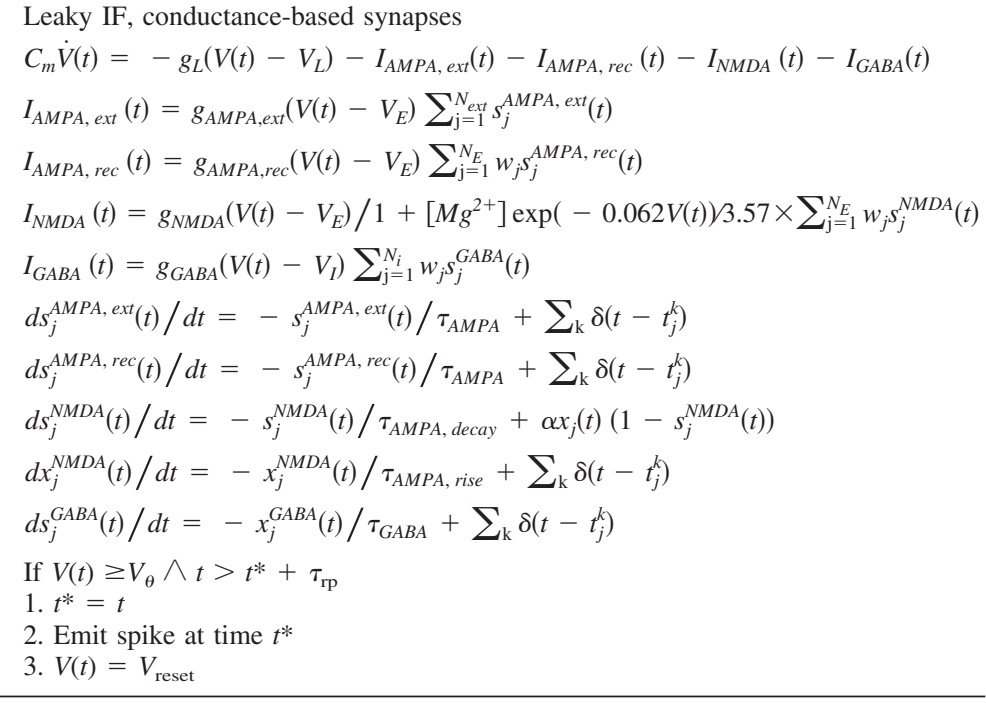

D. Input
Type

Poisson generator
Description

Fixed rate, $N_{\mathrm{ext}}$ Poisson generators per neuron; each one projects to one neuron

E. Measurements

Spike activity; firing rates calculated using spike count in a $50 \mathrm{~ms}$ time window shifted by $5 \mathrm{~ms}$ step

Model is summarized in section $A$ and detailed in sections $B-E$. Parameter values are given in Table 1 .

network, it loses the competition with LC. The confidence network in this case makes the second decision, probabilistically as before, to have a lack of confidence in the first decision, in that the LC population wins the competition. The confidence decision network thus acts as a decision-making network to make confident decisions if the firing rates from the first, decision-making, network are high and to make lack of confidence decisions if the firing rates from the first, decisionmaking, network are low.

On trials when the (first) decision network is correct, the input to the $\mathrm{C}$ population coming from the $\mathrm{DA}$ and $\mathrm{DB}$ neurons increases as a function of $\Delta \lambda$ and the $\mathrm{C}$ pool tends to win the competition more frequently (see Fig. 3). Thus a decision to act confidently about one's first decision is more likely to be made as $\Delta \lambda$ increases on correct trials. On the other hand, when the first network makes an error, the $\mathrm{C}$ population tends to win the competition less frequently as $\Delta \lambda$ increases, as shown in Fig. 3, and correspondingly on error trials the proportion of trials on which the LC population wins increases with $\Delta \lambda$. (The percentage correct of the LC population is the complement of that shown in Fig. 3.) Thus a decision to lack confidence about one's first decision is more likely to be made as $\Delta \lambda$ increases 


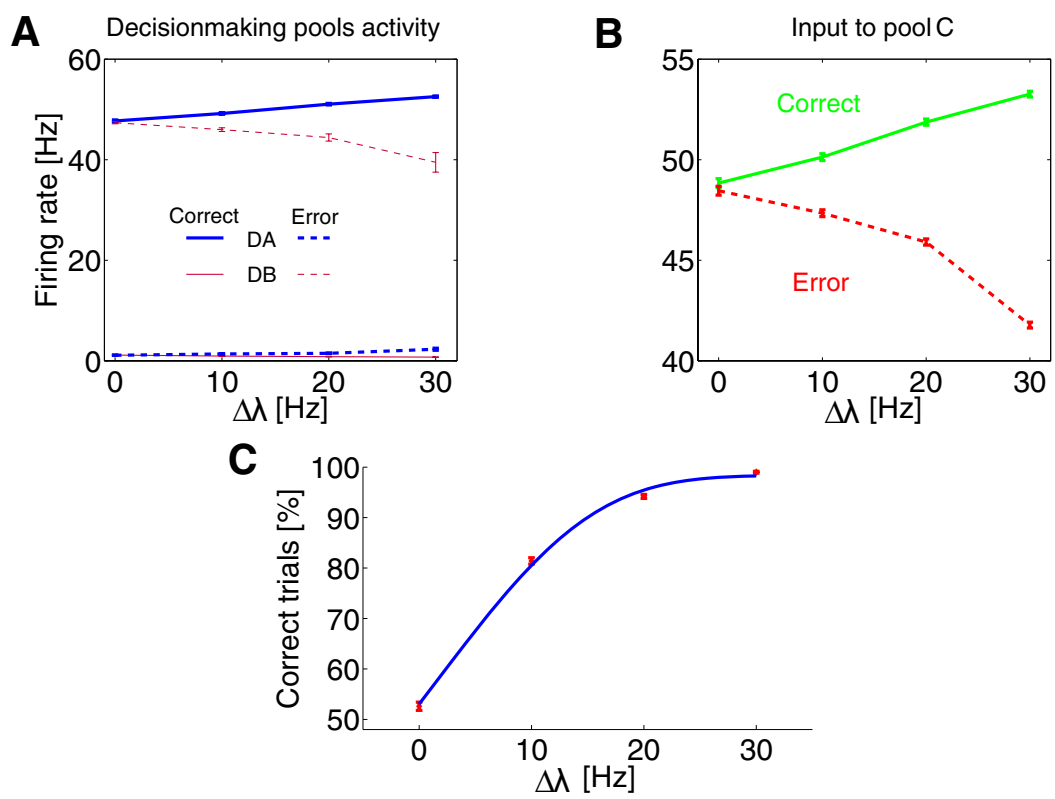

FIG. 2. Performance of the decision-making (first) network. $A$ : when pool DA correctly wins the competition, its firing rates are high and increase as a function of $\Delta \lambda$. When pool DB incorrectly wins, making an error due to the noise, and has a high firing rate, its firing rate decreases as a function of $\Delta \lambda$. This modulation of the firing rates by $\Delta \lambda$ was also observed experimentally (personal communication from Z. Mainen). The low firing rates when $\mathrm{DB}$ loses the competition on correct trials and when DA loses the competition on error trials are also shown. The error bars represent the SE. (The numbers of correct trials were between 524 and 990 and the numbers of error trials from 52 to 472 .) Confidence is thus encoded in the firing rates of the winning attractor and is an emergent property of the decisionmaking network. The firing rates were calculated averaging over trials the activity of neurons in the last second of each trial, from time $t=2,000 \mathrm{~ms}$ to $t=3,000 \mathrm{~ms}$. $B$ : sum of the firing rates from the DA and DB populations as a function of $\Delta \lambda$. This provides the input to the confidence (second) network selective pool $\mathrm{C}$. The error bars show the SE. $C$ : the percentage correct performance of the decision-making network as a function of $\Delta \lambda$. (The error bars were estimated from the binomial distribution and were small. The points are fitted by a Weibull function.)

on error trials and this might make one abort such a trial, as in the experiment of Kepecs et al. (2008).

The general time structure of the neuronal activity in the model is in qualitative accordance with the experimental results (Kepecs et al. 2008). As shown in Fig. 4, the confidence decision takes place after the first decision and separation of the firing rates of the two selective populations $\mathrm{C}$ and LC occurs after the decision-making network has reached a decision state, as in Fig. 3, $A-D$ of Kepecs et al. (2008).

It is important to examine the firing rates in the $\mathrm{C}$ and the $\mathrm{LC}$ attractor neuronal populations as a function of $\Delta \lambda$ on correct and incorrect trials because they provide an account for neuronal responses recorded during decision making (Kepecs et al. 2008) and those neurophysiological results in turn validate the

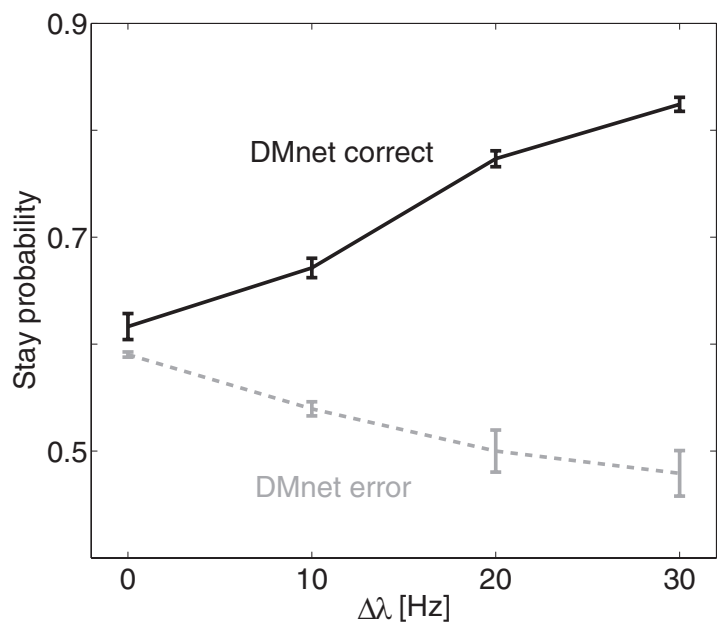

FIG. 3. Performance of the confidence decision (second) network. The proportion of trials on which in the second network the Confidence (C) population won the competition as a function of $\Delta \lambda$ for trials on which the decision-making (first) network (DMnet) was correct or incorrect. The performance of the LC population was the complement of this. [The parameters were set so that with $\Delta \lambda$ close to 0 , roughly $60 \%$ of the trials were $\mathrm{C}$ trials, to be qualitatively in the same direction as in the experimental findings of Kepecs et al. (2008).] model. We find for the confidence decision-making network that on correct trials with high $\Delta \lambda=30$ (easy perceptual decisions), $\mathrm{C}$ has a high firing rate, whereas it has a lower rate for $\Delta \lambda=10$ (i.e, difficult decisions), as shown in Fig. 5. Conversely, on error trials when the firing rates in the firstlevel, decision-making, network are lower, the confidence neurons $\mathrm{C}$ lose the competition and have relatively low firing rates, which decrease even further as the magnitude of $\Delta \lambda$ increases.

The firing rates (mean) in the confidence decision-making network of the $\mathrm{C}$ (confident, "positive outcome") and LC (lack of confidence, "negative outcome") populations of neurons for trials when the first decision-making network is correct or incorrect as a function of $\Delta \lambda$ are shown in Fig. 6 . The thick lines show the mean firing rates for the $\mathrm{C}$ and LC pools for all trials on which the first network was correct or in error. We identify the LC population of neurons with the negative outcome population of neurons described by Kepecs et al. (2008), which have similar properties, as shown in Supplemental Fig. $\mathrm{S} 1$. Further, we identify the $\mathrm{C}$ population of neurons with the positive outcome population of neurons described by Kepecs et al. (2008), which have similar properties, as shown in Supplemental Fig. S1.

However, as shown in Fig. 3, the confidence decisionmaking network was itself increasingly incorrect [i.e., took a confidence decision that was inconsistent with the decision made by the (first) decision-making network] as $\Delta \lambda$ approached 0 and the firing rates in the thick lines of Fig. 6 reflect the fact that on some trials the $\mathrm{C}$ pool won the competition and on some trials it lost. This is effectively how Kepecs et al. (2008) presented their data (for they knew only whether the first-stage decision itself was correct and did not measure while recording whether the rat took a confidence-related decision to stay with or abort a trial) and there is good correspondence, as can be seen by comparing Supplemental Fig. S1 with Fig. 3. If instead of taking the mean firing rate of the $\mathrm{C}$ neurons based only on whether the first decision was correct, we take just the trials on which the $\mathrm{C}$ (confidence) pool won the competition, the thin lines of Fig. 6 show for the $\mathrm{C}$ pool an average rate of 


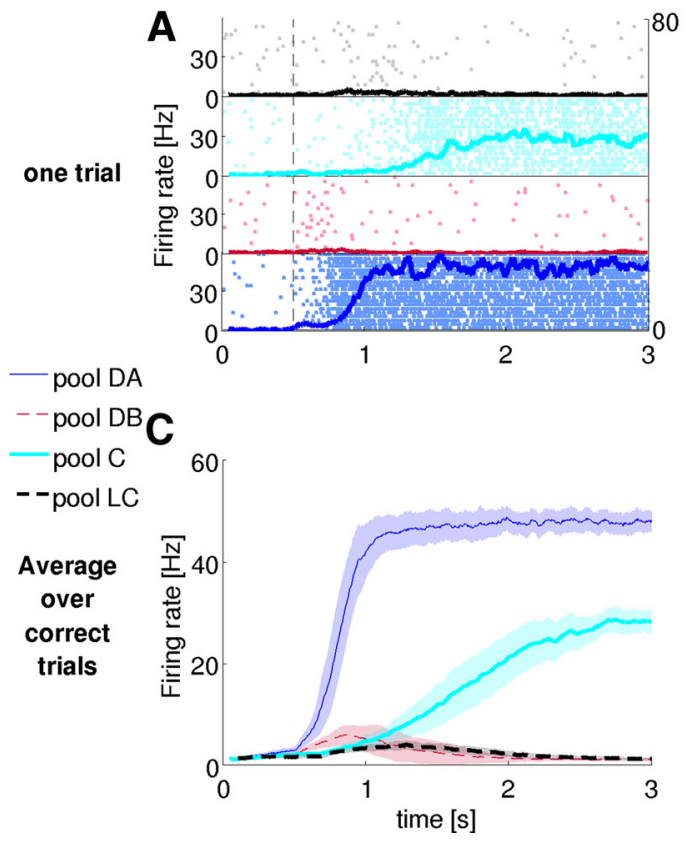

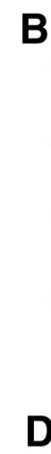

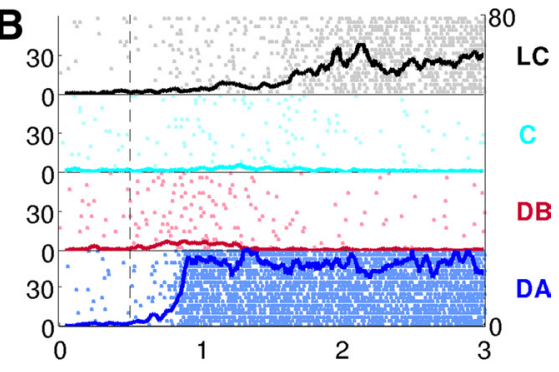

D

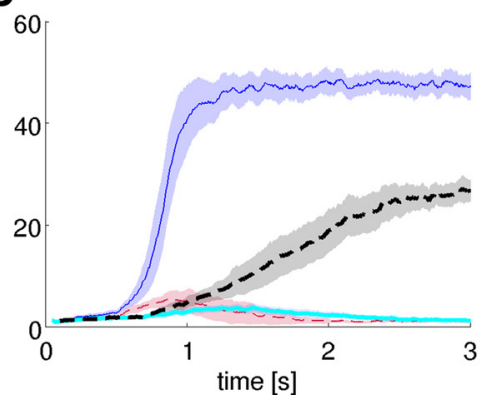

FIG. 4. Examples of the time courses of the neuronal activity in the selective pools of the decision-making (first) network and of the confidence decision (second) network for a decision at chance $(\Delta \lambda=0)$. $A$ and $B$ : the activity in time of selective pools in the decision-making network and in the confidence network. Raster plots show the activity of 20 sample neurons for each selective population in one trial (dashed vertical lines mark stimulus onset). Superimposed lines show the average firing rates for that trial. On $60 \%$ of trials the confidence network selective pool $\mathrm{C}$ won the competition $(A)$. On $40 \%$ of trials pool LC won the competition (B). $C$ and $D$ : the average firing rates over all correct trials for the same conditions, respectively, as $A$ and $B$. The separation of the firing rates begins after the decision is made and the general temporal structure of the network is in qualitative accordance with the experimental results of Kepecs et al. (2008). close to 28 spikes/s that tends to increase with $\Delta \lambda$ when the first network is correct and tends to decrease with $\Delta \lambda$ when the first network is in error. (This is supported by the data shown in Fig. 5.) If we take just the trials on which the $C$ population lost the competition, the thin lines show for the $\mathrm{C}$ pool an average rate of close to 2 spikes/s. Conversely, for the LC pool of neurons, if we take just the trials on which the LC population won the competition, the thin lines show for the LC pool an average rate of close to 26 spikes/s. If we take just the trials on which the LC population lost the competition, the thin lines show for this LC pool an average rate of close to 2 spikes/s. These firing rates shown in the thin lines in Fig. 6 are generally as expected and the differences with $\Delta \lambda$ are due to whether the output of the decision-making (first) network shown in Fig. $2 B$ is consistent or inconsistent with the decision made by the confidence decision (second) network, which is of course influenced by the spiking noise in the confidence decision network, which can make the wrong decision given the evidence it receives from the decision-making network shown in Fig. $2 B$.

\section{I S C U S S I O N}

We have shown how decision confidence is an emergent property of a neurophysiologically based decision-making pro- cess and is encoded in a graded way by the continuously graded firing rates of the neurons in an integrate-and-fire attractor decision-making network. (This is shown by the results for the first decision-making network.) We have also shown how within this neurobiologically based framework for decision making, two separate networks are essential for the ability to make a decision involving a choice about one's confidence in a prior decision. We have also shown how the model is confirmed by and provides a computational account for the neurophysiological findings of Kepecs et al. (2008) (see Supplemental Fig. S1) and also provides a new interpretation of the data recorded by Kepecs et al. (2008), as described in more detail in the following text. We also make new predictions about the types of neuronal response that will be found when a confidence-based decision must be made, as subsequently described.

The fact that the changes of firing rates found in the rat by Kepecs et al. (2008), as shown in Supplemental Fig. S1 as a function of $\Delta \lambda$, are comparable with those shown in the thick lines in Fig. 6 provides good support for the present model. However, Kepecs et al. (2008) did not distinguish trials in which a second-layer confidence decision network was in error or not because they did not record neuronal activity when they could examine whether the rat aborted a trial-we suggest that

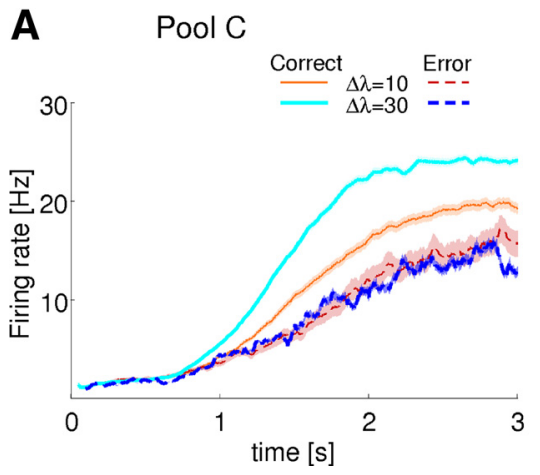

B Pool LC

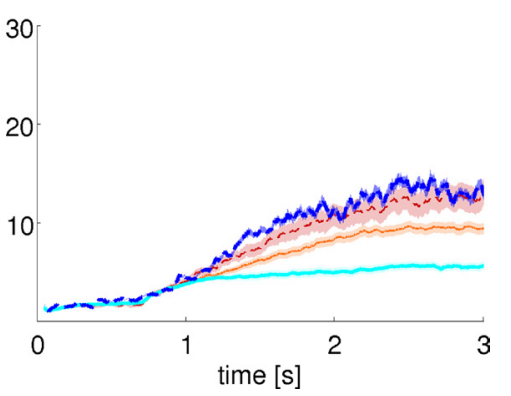

FIG. 5. Firing rates in the confidence decision-making network of the $\mathrm{C}$ (confident) and LC (lack of confidence) populations of neurons for trials when the first decision-making network is correct or incorrect for easy decisions $(\Delta \lambda=30)$ and difficult decisions $(\Delta \lambda=10)$. The firing rates shown are averaged merging together confident and lack of confidence trials. The same mixture is shown in thick lines in Fig. 6, to show the correspondence with the experimental results. (The shaded areas represent the SE. The numbers of trials are in the range from 72 to 990.) The decision cues were turned on at $t=$ $500 \mathrm{~ms}$. 

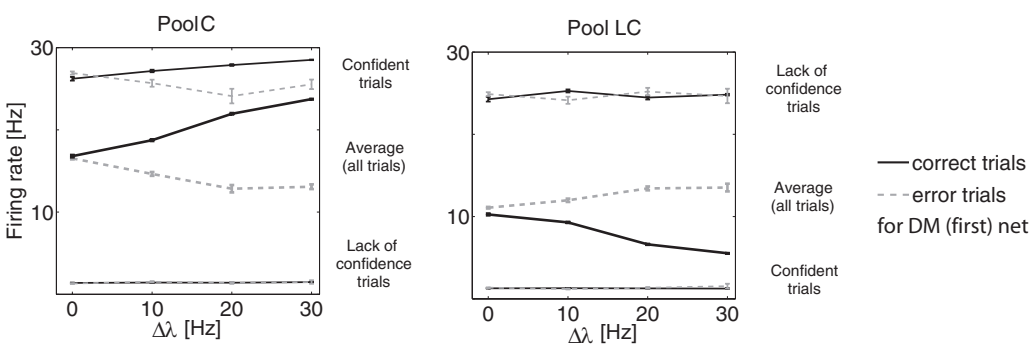

FIG. 6. Firing rates (mean \pm SE) in the confidence decision-making network of the $C$ (confident, "positive outcome") and LC (lack of confidence, "negative outcome") populations of neurons. Labels "Confident trials" and "Lack of confidence trials" mark, respectively, trials on which the C (confidence) population and LC (lack of confidence) population won the competition. The activities of the $\mathrm{C}$ and LC populations of neurons, averaged over all trials, confident and lack of confidence, are shown with label "Average (all trials)." As shown in Fig. 3, the confidence decision-making network was itself increasingly incorrect as $\Delta \lambda$ approached 0 and the firing rates in the thick lines reflect the fact that on some trials the $\mathrm{C}$ population won the competition, and on some trials it lost. (There is no correct/error distinction for trials with $\Delta \lambda=0$. For illustration purposes, trials in which the final choice was A were labeled as correct trials and trials in the choice was B were labeled as error trials.) The firing rates shown were calculated by averaging the activity of neurons in the last second of each trial, from time $t=2,000 \mathrm{~ms}$ to $t=3,000 \mathrm{~ms}$. [The error bars for the thin lines (confidence and lack of confidence decision trials) represent the SE and the number of trials was in the range 28 to 760 , with few error trials occurring with high values of $\Delta \lambda$.]

it would now be interesting to do this. Further, it is notable that the change of firing rate with $\Delta \lambda$ found in the rat matches only that of the thick lines in Fig. 6, which includes all trials irrespective of the decision made by the confidence decision (second) network and not by the thin lines in Fig. 6, which reflect the decision made by the confidence network. This leads to the novel prediction that results will be found different from those presented by Kepecs et al. (2008) if in a future experiment the responses of similar neurons are separated according to whether each trial is aborted. We predict in particular that the neurons will have activity like that shown in the thin lines in Fig. 6 and will be of two types. One type will be similar to that of the $\mathrm{C}$ (confident in the prior decision) neurons shown in Fig. 6 in which the firing rate is 1 ) high on trials on which the confidence decision is to stay with the first decision and 2) low if the confidence (second) decision is to abort the trial. The prediction further is that the firing rates of these confidence neurons will change with $\Delta \lambda$, as shown by the thin lines in Fig. $6 \mathrm{~A}$; that is, these high firing rates will tend to increase as a function of $\Delta \lambda$ if the first decision (made by the decisionmaking, first, network) is consistent with the evidence (i.e., correct), as shown at the top of Fig. $6 A$, and to decrease as a function of $\Delta \lambda$ if the first decision (made by the decisionmaking, first, network) is inconsistent with the evidence (i.e., is an error), as also shown at the top of Fig. $3 A$. The second type of neuron will be similar to that of the LC (lack of confidence in the prior decision) neurons shown in Fig. $6 B$, in which the firing rate is high on individual trials on which the confidence decision is to abort the trial and low if the confidence (second) decision is to stay with the first decision. (The firing rates of the LC population do not change much with $\Delta \lambda$, as shown by the thin lines in Fig. $6 B$, because the input from the saturating neurons has a fixed firing rate.) It is only when we categorize the neurons according to whether the first decision was correct that curves similar to those shown by thick lines in Fig. 6 and, as reported by Kepecs et al. (2008), will be found and such curves and analyses do not fully capture the properties of the confidence decision-related neurons, which are as shown in the thin lines in Fig. 6.

We used the model to account for neural data recorded in the OFC by Kepecs et al. (2008). However, different brain areas are involved in different types of decision making (Hernandez et al. 2002; Kim and Shadlen 1999; Rolls and Deco 2010; Romo et al. 2002) and it is accordingly plausible that other brain areas can process confidence-related information. Therefore we tried to keep the model as simple as possible to propose a generic mechanism for confidence-related representation and decision making, which is not only consistent with and provides an interpretation of experimental data from Kepecs et al. (2008), but also provides a generic account of confidencerelated decision making in other brain areas.

Our model is designed based on a binary decision-making task, but it could also be slightly modified to encompass multiple-choice decision making. In some recent work the theoretical framework of biased competition that we adopted has been developed to account for multiple-choice decision making (Albantakis and Deco 2009; Furman and Wang 2008). A possible extension of our model would be a combination of an architecture like the one proposed by Albantakis and Deco (2009), but with a second confidence network strongly connected with the decision neurons. We propose that such an extended model based on our idea can also account for decisions based on confidence in multiple-choice decision-making processes.

The architecture of our model is based on two layers: one for the perceptual decision and one to monitor the confidence level of the first decision. However, there is no restriction to two layers and deeper architectures could be built to perform more complex functions. In fact, the second layer also undertakes a decision process and thus a third layer could monitor the activity of the second layer. The proposed mechanism could thus be extended to account for nested monitoring functions (cf. Hofstadter 2007). Although no natural restriction is imposed on the mechanism and learning could shape a nested network hierarchy, a second-order confidence-related decision (and eventually a third-order) may be of less use than a judgment about a first-level (e.g., perceptual) decision.

The confidence decision (second) network is in effect monitoring the decisions made by the first network and can cause a change of behavior, choosing to abort the trial, if the second network's assessment of the decision made by the first network is that the first decision is not a confident decision. Now this is the type of description and language used to describe "monitoring" functions, taken to be high-level cognitive processes, possibly related to consciousness (Block 1995; Lycan 1997). For example, in an experiment performed by Hampton (2001) (experiment 3 ), a monkey performing a short-term memory 
task could choose an "escape flag" to start another trial. With longer delays, when memory strength might be lower partly due to noise in the system and confidence therefore might be lower, the monkey was more likely to choose the escape flag. The experiment is described as showing that the monkey is thinking about his own memory, which is a case of metamemory that may be related to consciousness (Heyes 2008). However, the decision about whether to escape from a trial can be made just by adding a second decision network to the first decision network. Thus we can account for what seem like complex cognitive phenomena with a simple system of two attractor decision-making networks (Fig. 1). The design of Kepecs et al. (2008) was analogous, in that the rat could choose to abort a trial if decision confidence was low and, again, this functionality can be implemented by two attractor decisionmaking networks, as described here.

There are other more complex types of "self-monitoring," such as that described as occurring in a commentary that might be based on reflection on previous events and appears to be more closely related to consciousness (Rolls 2007; Weiskrantz 1997). Our aim was not to account for such complex monitoring functions. Rather we claim that some types of "selfmonitoring" are computationally simple and the model we propose can be a building block for a better understanding of such a high-level cognitive function.

\section{A C K N O W L E D G M E N T S}

This work was supported by the European Community's Seventh Framework Programme FP7/2007-2013 under grant agreement number 214728-2; by Spanish Research Projects BFU2007-61710/BFI and CONSOLIDER INGENIO 2010; by the Oxford McDonnell Centre for Cognitive Neuroscience; and by the Oxford Centre for Computational Neuroscience.

\section{I S C L O S URES}

No conflicts of interest, financial or otherwise, are declared by the author(s).

\section{R E F EREN C ES}

Abeles A. Corticonics. New York: Cambridge Univ. Press, 1991.

Albantakis L, Deco G. The encoding of alternatives in multiple-choice decision making. Proc Natl Acad Sci USA 106: 10308-10313, 2009.

Block N. On a confusion about a function of consciousness. Behav Brain Sci 18: 22-47, 1995.

Brody C, Hernandez A, Zainos A, Romo R. Timing and neural encoding of somatosensory parametric working memory in macaque prefrontal cortex. Cereb Cortex 13: 1196-1207, 2003.

Brunel N, Wang X-J. Effects of neuromodulation in a cortical network model of object working memory dominated by recurrent inhibition. J Comput Neurosci 11: 63-85, 2001.

Brunel N, Wang X-J. What determines the frequency of fast network oscillations with irregular neural discharges? I. Synaptic dynamics and excitation-inhibition balance. J Neurophysiol 90: 415-430, 2003.

Dayan P, Abbott LF. Theoretical Neuroscience. Cambridge, MA: MIT Press, 2001

Deco G, Marti D. Deterministic analysis of stochastic bifurcations in multistable neurodynamical systems. Biol Cybern 96: 487-496, 2007.

Deco G, Rolls ET. A neurophysiological model of decision-making and Weber's law. Eur J Neurosci 24: 901-916, 2006.

Deco G, Rolls ET, Romo R. Stochastic dynamics as a principle of brain function. Prog Neurobiol 88: 1-16, 2009.
Felsen G, Mainen Z. Motor planning in the rat superior colliculus. Frontier in Systems Neuroscience. Conference Abstract: Computational and systems neuroscience, 2009. doi:10.3389/conf.neuro.06.2009.03.176.

Furman M, Wang X-J. Similarity effect and optimal control of multiplechoice decision making. Neuron 60: 1153-1168, 2008.

Gold JI, Shadlen MN. The neural basis of decision making. Annu Rev Neurosci 30: 535-574, 2007.

Hampton RR. Rhesus monkeys know when they can remember. Proc Natl Acad Sci USA 98: 5539-5362, 2001.

Hernandez A, Zainos A, Romo R. Temporal evolution of a decision-making process in medial premotor cortex. Neuron 33: 959-972, 2002.

Heyes C. Beast machines? Questions of animal consciousness. In: Frontiers of Consciousness, edited by Weiskrantz L, Davies M. Oxford, UK: Oxford Univ. Press, 2008, p. 259-274.

Hofstadter DR. I Am a Strange Loop. New York: Basic Books, 2007.

Jonsson FU, Olsson H, Olsson J. Odor emotionality affects the confidence in odor naming. Chem Senses 30: 29-35, 2005.

Kepecs A, Uchida N, Zariwala HA, Mainen F. Neural correlates, computation and behavioural impact of decision confidence. Nature 455: 227-231, 2008.

Kiani R, Shadlen MN. Representation of confidence associated with a decision by neurons in the parietal cortex. Science 324: 759-764, 2009.

Kim J, Shadlen M. Neural correlates of a decision in the dorsolateral prefrontal cortex of the macaque. Nat Neurosci 2: 176-185, 1999.

Lycan WG. Consciousness as internal monitoring. In: The Nature of Consciousness: Philosophical Debates, edited by Block N, Flanagan O, Guzeldere G. Cambridge, MA: MIT Press, 1997, p. 755-771.

Machens C, Romo R, Brody C. Flexible control of mutual inhibition: a neural model of two-interval discrimination. Science 307: 1121-1124, 2005.

Marti D, Deco G, Del Giudice P, Mattia M. Reward-biased probabilistic decision-making: mean-field predictions and spiking simulations. Neurocomputing 39: 1175-1178, 2006.

Marti D, Deco G, Mattia M, Gigante G, Del Giudice P. A fluctuation-driven mechanism for slow decision processes in reverberant networks. PLOS ONE 3: e2534, 2008.

Mattia M, Del Giudice P. Population dynamics of interacting spiking neurons. Phys Rev E 66: 051917, 2002.

Mattia M, Del Giudice P. A distribution of spike transmission delays affects the stability of interacting spiking neurons. Sci Math Japon 18: 335-342, 2003.

Mattia M, Del Giudice P. Finite-size dynamics of inhibitory and excitatory interacting spiking neurons. Phys Rev E 70: 052903, 2004.

Ratcliff R, Rouder JF. Modeling response times for two-choice decisions. Psychol Sci 9: 347-356, 1998.

Ratcliff R, Zandt TV, McKoon G. Connectionist and diffusion models of reaction time. Psychol Rev 106: 261-300, 1999.

Rolls ET. A computational neuroscience approach to consciousness. Neural Networks 20: 962-982, 2007.

Rolls ET. Memory, Attention, and Decision-Making. A Unifying Computational Neuroscience Approach. Oxford, UK: Oxford Univ. Press, 2008.

Rolls ET, Deco G. The Noisy Brain: Stochastic Dynamics as a Principle of Brain Function. Oxford, UK: Oxford Univ. Press, 2010.

Romo R, Hernandez A, Zainos A, Lemus L, Brody C. Neural correlates of decision-making in secondary somatosensory cortex. Nat Neurosci 5: $1217-$ $1225,2002$.

Usher M, McClelland J. On the time course of perceptual choice: the leaky competing accumulator model. Psychol Rev 108: 550-592, 2001.

Vickers D. Decision Processes in Visual Perception. New York: Academic Press, 1979.

Vickers D, Packer J. Effects of alternating set for speed or accuracy on response time, accuracy and confidence in a unidimensional discrimination task. Acta Psychol 50: 179-197, 1982.

Wang XJ. Synaptic basis of cortical persistent activity: the importance of NMDA receptors to working memory. J Neurosci 19: 9587-9603, 1999.

Wang XJ. Probabilistic decision making by slow reverberation in cortical circuits. Neuron 36: 955-968, 2002.

Weiskrantz L. Consciousness Lost and Found. Oxford, UK: Oxford Univ. Press, 1997. 